\title{
ABORDAGEM FISIOTERAPÊUTICA AO IDOSO NA ATENÇÃO PRIMÁRIA À SAÚDE: UMA REVISÃO INTEGRATIVA
}

\author{
PHYSIOTHERAPEUTIC APPROACH TO THE ELDERLY IN PRIMARY \\ HEALTHCARE: AN INTEGRATIVE REVIEW
}

Jerssica Mayara Agustinho da Silva ${ }^{1}$

Thainá Rayane Bezerra Lemos²

\begin{abstract}
RESUMO: OBJETIVO: Identificar a abordagem fisioterapeuta do idoso na Atenção Primária à saúde. MÉTODOS: O presente estudo trata-se de uma pesquisa integrativa da literatura. A coleta de dados se deu pela Biblioteca virtual em saúde, no período de dezembro a fevereiro de 2021 com os seguintes descritores "Fisioterapia" AND "Idoso" AND "Fisioterapia" AND "Atenção Primária à Saúde" RESULTADOS: Foram encontrados 54 artigos científicos do ano de 2015 a 2021 com texto completo disponível e após os critérios de exclusão e inclusão 05 artigos foram analisados. Os profissionais precisam estar atentos para receber e orientar os pacientes, nesse sentido, o NúcleoAmpliado de Saúde da Família e Atenção Básica faz parte do primeiro contato, onde o Fisioterapeuta atua com estratégias de prevenção e reabilitação para os idosos, o que evidencia sua importância na reabilitação junto com a equipe da Atenção primaria traz inúmeros benefícios para esses usuarios. CONCLUSÃO: Diante deste contexto, o fisioterapeuta é capacitado para realizar uma reabilitação resolutiva de acordo com cada individualidade do paciente idoso na atenção primaria, este faz a diferença, pois conta com a equipe multiprofissional para dar um proposito frutifero para as questões de saúde trazidas pelos usuários.
\end{abstract}

Palavras chave: Reabilitação. Idoso. Atenção primária à saúde.

\footnotetext{
1 Fisioterapeuta, graduada pela Universidade Federal da Paraíba (UFPB); Residente no Programa de Residência Multiprofissional em Saúde da Família e Comunidade (Secretaria Municipal de Saúde João Pessoa/Faculdade de Ciências Médicas); Pós-graduada Terapia Intensiva em Fisioterapia Centro Universitário de João Pessoa (UNIPÊ). E-mail: Jerssica.mayara@gmail.com.

${ }^{2}$ Fisioterapeuta, graduada pela Universidade Federal da Paraíba (UFPB); Residente no Programa de Residência Multiprofissional em Saúde da Família e Comunidade (Secretaria Municipal de Saúde João Pessoa/Faculdade de Ciências Médicas). E-mail:thainavieria17@hotmail.com.
} 
ABSTRACT: OBJECTIVE: To identify the physiotherapistapproach of the elderly in Primary Health Care. METHODS: This study is an integrative literature search. Data collection took place through the Virtual Health Library, from Decemberto February 2021 with the following descriptors "Physiotherapy" AND "Elderly" AND "Physiotherapy" AND "Primary Health Care" RESULTS: 54 scientific articles from year from 2015 to 2021 with full text available and after the exclusion and inclusion criteria 05 articles were analyzed. Professionals need to be attentive to receive and guide patients, in this sense, the Extended Family Health and Primary Care Center is part of the first contact, where the Physiotherapist works with prevention and rehabilitation strategies for the elderly, which showsits importance in rehabilitation together with the Primary Care team brings numerous benefits to these users. CONCLUSION: In this context, the physiotherapist is trained to perform a resolutive rehabilitation according to each individuality of the elderly patient in primary care, this makes a difference, as it relies on the multiprofessional team to provide a fruitful purpose for health issues brought by users.

Keywords: Rehabilitation. Elderly. Primary Health Care. 


\section{INTRODUÇÃO}

A atenção Primária à Saúde (APS) se constitui como a principal porta de entrada no Sistema Único de Saúde (SUS), e seu foco está nas doenças crônicas não transmissíveis. Por conseguinte, seu maior público é a população idosa (PLACIDELI., et al 2020).

O fisioterapeuta atua na APS e seu plano de tratamento aborda na reabilitação e prevenção a disfunções que possam acometer a saúde do idoso. Suas atividades são variadas podendo ser em grupo e individuais, sendo a avaliação um passo significativo para proporcionar uma conduta eficaz e adequada ao idoso (CARDOSO; DE MELLO, 2020).

As atividades em grupo podem ser desenvolvidas nas Academias da Saúde, vinculadas ou não a Unidade Básica de Saúde (UBS), onde as atividades visam uma conduta baseada em exercícios ativos livres como por exemplo alongamentos ativos, exercícios de fortalecimento de baixa intensidade e exercícios funcionais que auxiliem nas suas atividades devida diária (AVD) e funcionalidade (MONTENEGRO; BARBOSA; NOVAES, 2020).

Outro tipo de modalidade que auxilia na reabilitação do idoso são os atendimentos Home Care (Atendimentos Domiciliares) encontradas na Política do Serviço de Atendimento Domiciliar (SAD). O atendimento Home Care vai depender do que o paciente está precisando naquele momento e é realizado após uma avaliação minuciosa da doença que acomete o idoso (BRASIL 2013).

Existem diversas patologias e doenças crônicas que atingem a população idosa, sendo em grande parte acidente vascular encefálico (AVE), infarto agudo do miocárdio (IAM), neoplasias, osteoporose, diabetes, fraturas. Diante disto, as sequelas são inevitáveis na maioria dos casos, o que leva o idoso a um estado de fragilidade. Nesse sentido, o processo de reabilitação entra em cena, no qual o fisioterapeuta tem a capacidade de auxiliar neste momento, introduzindo uma conduta 
adequada para que o idoso possa recuperar sua funcionalidade e voltar a realizar as AVD (DOS SANTOS., et al 2019).

Nesta perspectiva, é válido enfatizar também que reabilitação do idoso na APS é considerada uma área que carrega constantes desafios para o fisioterapeuta, mas que é de fundamental importância para o idoso, a exemplo das visitas domiciliares que podem ser realizadas com a equipe multiprofissional. $O$ retorno às AVD é um dos objetivos principais na reabilitação, incluindo atividades simples como movimentar-se e caminhada ao redor da sua própria residência com o auxílio dos familiares, visando uma conduta mediadora na atenção primária (PIUVEZAM., et al 2016).

Diante do exposto, o fisioterapeuta tem um papel de fundamental importância na vida do idoso, proporcionando um impacto positivo no seu cotidiano. Sua conduta visa a prevenção, promoção e reabilitação da condição de saúde nesta fase de vida, para que se possa atingir uma vida não apenas duradoura, mas com melhor qualidade (DE LIMA., et al 2017). Com isso o objetivo deste estudo é identificar a abordagem fisioterapeuta do idoso na AtençãoPrimária à saúde.

\section{MÉTODOS}

O presente estudo trata-se de uma pesquisa integrativa da literatura, em sua estrutura está a agrupar e sintetizar resultados de um determinado problema de pesquisa finalizando com suas possíveis previsões de novas pesquisas. Sua metodologia aborda seis etapas: identificação do tema e pergunta norteadora do estudo; seleção da amostra; categorização dos estudos selecionados; aplicação dos estudos incluídos na revisão; interpretação e discussão dos resultados e apresentação final da revisão integrativa (MEDEIROS; PAIXÃO; MIRANDA, 2017).

Sendo assim, a pergunta para este estudo foi: Como está sendo realizada a fisioterapia ao idoso na Atenção Primária à Saúde? A coleta de dados se deu pela Biblioteca Virtual em Saúde (BVS), no período de dezembro de 2020 a fevereiro de 2021. A primeira parte da pesquisa foi a identificação dos descritores em ciências da 
saúde (DeCS), os mais relevantes para o tema proposto foram: "Reabilitação", "Idoso" e "Atenção Primária à Saúde".

Concomitantemente, foi realizado o cruzamento entre os DeSC pelo Boleano AND ("Fisioterapia" AND "Idoso" AND "Fisioterapia" AND "Atenção Primária à Saúde"). Foram encontrados 54 artigos com texto completo disponível entre os anos 2015 a 2021. Ser em formato de tese, monografia, dissertações, textos não científicos e artigos que apresentaram erroao acesso caracterizaram aos critérios de exclusão. Assim, apenas cinco artigos atenderam ao critério de inclusão. A figura a seguir apresenta a sequência de busca e eliminação dos artigos que estava condito ao tema proposto.

Figura 1 - Sequência de busca e eliminação dos artigos.

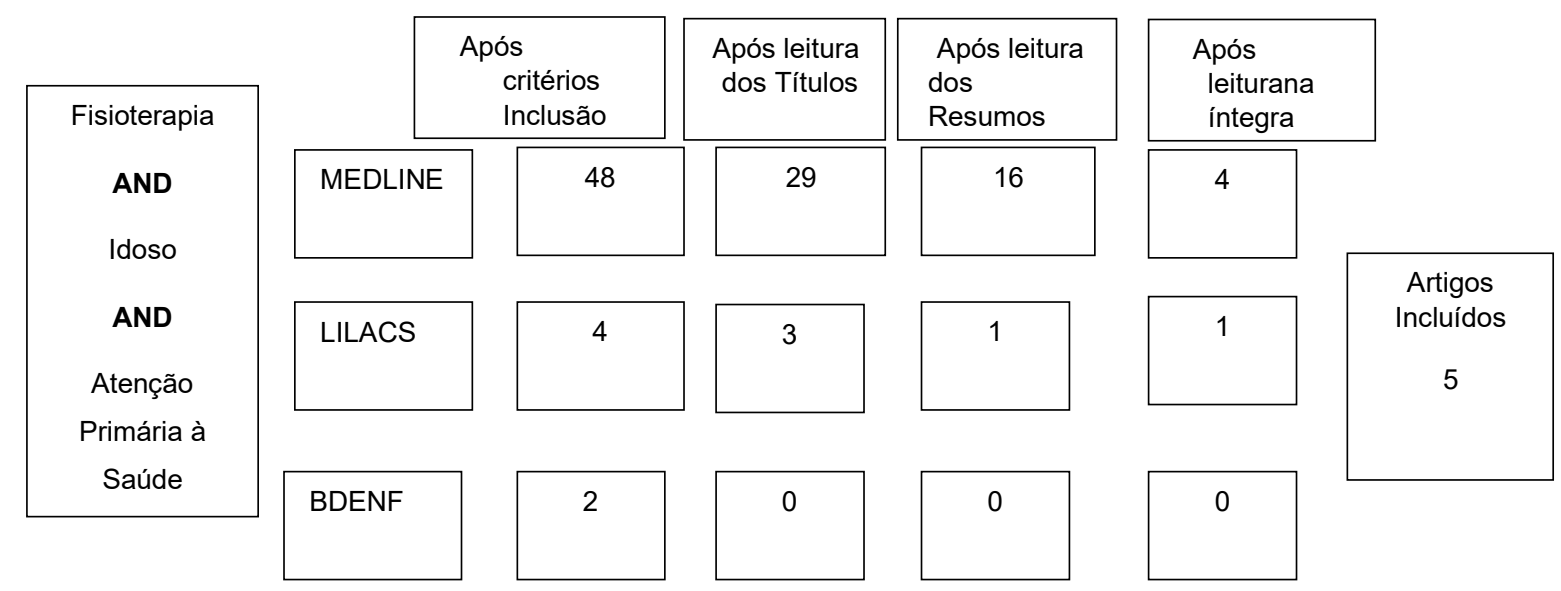




\section{RESULTADOS}

Tabela 1 - Descrição dos artigos contendo auto, ano, título e periódico.

\begin{tabular}{|c|c|c|c|}
\hline AUTOR & ANO & PERIÓDICO & COD \\
\hline ØSTERÅS, N. et al. & 2019 & $\begin{array}{l}\text { Implementing a structured model for } \\
\text { osteoarthritis care in primary healthcare: A PLos Medicine } \\
\text { stepped-wedge cluster-randomised trial. }\end{array}$ & A1 \\
\hline $\begin{array}{c}\text { TOMASIS, A. V. R. } \\
\text { et al. }\end{array}$ & 2017 & $\begin{array}{l}\text { Urinary incontinence in elderly people: care } \text { Texto \& } \\
\text { practices and care proposal in primary healthcontextoenferm } \\
\text { care } \\
\text { [Internet] }\end{array}$ & A2 \\
\hline $\begin{array}{l}\text { DÍAZ-CERRILLO, J. } \\
\text { L. et al. }\end{array}$ & 2016 & $\begin{array}{l}\text { Ensayo no aleatorizado de una intervención } \\
\text { educativa basada en principios cognitivo- } \\
\text { conductuales para pacientes con lumbalgia } \\
\text { crónica inespecífica atendidos en fisioterapia de } \\
\text { atención primaria. }\end{array}$ & B1 \\
\hline KINGE, J. M. et al. & 2015 & $\begin{array}{l}\text { Musculoskeletal disorders in Norway: } \\
\begin{array}{l}\text { BMC } \\
\text { prevalence of chronicity and use of primary andmusculoskeletal } \\
\text { specialist health care services. }\end{array} \\
\text { disorders }\end{array}$ & A1 \\
\hline $\begin{array}{c}\text { ANDERSSON, S. F. } \\
\text { et al. }\end{array}$ & 2017 & $\begin{array}{l}\text { Arthritis management in primary care-A study of } \\
\text { physiotherapists' current practice, educational usculoskeletal } \\
\text { needs and adherence to national guidelines. }\end{array}$ & B2 \\
\hline
\end{tabular}

Tabela 2 - Objetivos, tipo de estudo e desfecho dos artigos.

\begin{tabular}{|c|c|c|c|}
\hline OBJETIVOS & $\begin{array}{l}\text { TIPO DE } \\
\text { ESTUDO }\end{array}$ & DESFECHO & COD \\
\hline $\begin{array}{l}\text { Avaliar a eficácia desse } \\
\text { modelo naatenção primária. }\end{array}$ & $\begin{array}{l}\text { Ensaio clínicc } \\
\text { andomizado }\end{array}$ & $\begin{array}{l}\text { O estudo traz propostas de intervenção para } \\
\text { melhorar o modelo de atendimento a pessoas } \\
\text { doenças crônicas na atenção primaria em que } \\
\text { oseu público alvo está idosos participantes da } \\
\text { atenção primaria à saúde, em suma o estudo } \\
\text { apresentou resultados satisfatórios com adesão } \\
\text { positiva ao tratamento melhorando assim a } \\
\text { qualidade de vida. }\end{array}$ & A1 \\
\hline $\begin{array}{l}\text { Identificar o conhecimento e } \\
\text { as práticas assistenciais } \\
\text { sobre a incontinência } \\
\text { urinária em mulheres idosas } \\
\text { e desenvolver proposta de } \\
\text { cuidado a essas mulheres } \\
\text { para a promoção da saúde } \\
\text { no âmbito da atenção } \\
\text { primária de saúde. }\end{array}$ & Qualitativa & $\begin{array}{l}\text { A pesquisa envolveu processos de apreensão, } \\
\text { A2 síntese, teorização e transferência, fazendo } \\
\text { emergir dois eixos temáticos: percepção dos } \\
\text { profissionais acerca da incontinência urinária } \\
\text { em mulheres idosas; e a concepção de uma } \\
\text { proposta de cuidado à mulher idosa com } \\
\text { incontinência urinária. Constatou-se o pouco } \\
\text { conhecimento dos profissionais acerca da } \\
\text { incontinência urinária, bem como a falta de } \\
\text { informação em relação às possibilidades de } \\
\text { orientações para o autocuidado a respeito dos } \\
\text { sintomas urinários. }\end{array}$ & A2 \\
\hline
\end{tabular}




\begin{tabular}{|c|c|c|}
\hline $\begin{array}{l}\text { Valorar a influência de uma } \\
\text { intervenção educativa na } \\
\text { redução da "miedo- } \\
\text { evitación» e do } \\
\text { «catastrofismo al dolor» e na Ensaio quase } \\
\text { população com lombalgia experimental } \\
\text { crónica inespecífica atendida } \\
\text { na fisioterapia de atenção } \\
\text { primária. }\end{array}$ & $\begin{array}{l}\text { O estudo propõe estratégias de educação e } \\
\text { reabilitação para os pacientecom dor lombar } \\
\text { crônica que estão na Atenção Primária o } \\
\text { público em suma foram idosos supondo assim } \\
\text { que haja mais estudos para aprimorar esse } \\
\text { cuidado na atençãoprimária. }\end{array}$ & B1 \\
\hline $\begin{array}{l}\text { Estimar a prevalência de } \\
\text { distúrbios } \\
\text { musculoesqueléticos } \\
\text { crônicos e estimar a } \\
\text { prevalência de pessoas que Transversal } \\
\text { recebem serviços de saúde } \\
\text { primários e especializados } \\
\text { para esses distúrbios. }\end{array}$ & $\begin{array}{l}\text { O estudo apresenta o perfil de atendimento } \\
\text { mais procurados na atenção primária os mais } \\
\text { procurados médicos e fisioterapeutas. Com } \\
\text { prevalência do sexo feminine e com aumento } \\
\text { dos distúrbios musculoesquelético devido o } \\
\text { avanço da idade. Concluído um estudo que } \\
\text { evidencia a importância da fisioterapia na } \\
\text { procura em contexto da atenção primária à } \\
\text { saúde. }\end{array}$ & B1 \\
\hline $\begin{array}{l}\text { Investigar a prática } \\
\text { relacionada à artrite na } \\
\text { fisioterapia e } \\
\text { adesão estudar a a Transversal } \\
\text { baseados em evidências. }\end{array}$ & $\begin{array}{l}\text { O estudo traz em sua metodologia a } \\
\text { aplicação de B2 questionário para } \\
\text { fisioterapeutas daatenção primaria no contexto } \\
\text { do atendimento à pacientes com artrite } \\
\text { reumatoide, presumindo assim que são grupo } \\
\text { da população idosa que faz parte deste } \\
\text { contexto para o tratamento da fisioterapia na } \\
\text { atenção primaria. Os resultados mostraram se } \\
\text { satisfatórios em relação a conduta mediada por } \\
\text { esseprofissional na atenção primária. }\end{array}$ & B2 \\
\hline
\end{tabular}

\section{DISCUSSÃO}

A atenção Primária possui um leque de informações e ações para os usuários, sendo a porta de entrada do SUS. Suas ações visam reduzir as demandadas até a mais alta complexidadeatuado assim de forma preventiva, seu maior público sãos pessoas portadoras de doenças crônicas não transmissíveis, que por sua vez é a grande parte a população idosa (DEOLIVEIRA., et al 2017).

As doenças crônicas são enfermidades que na maioria das vezes representam um enfrentamento que perdurará pelo resto da vida, neste sentido a UBS tem estratégias de atuaçãopara lidar com esse público. A capacidade funcional destes usuários se encontra fragilizada e atividades de educação em saúde visa monitorar esses fatores para aprimorar a conduta mediadapela equipe (SILVA., et al 2019). 
A maioria das doenças tratadas na AP são a hipertensão arterial sistêmica (HAS) e a diabetes mellitus (DM) que, se descompensadas, podem desencadear disfunções em outras regiões do corpo podendo levar a complicações como, por exemplo, um acidente vascular encefálico (AVE) (TORRES., et al 2020).

Os profissionais precisam estar atentos para receber e orientar esses pacientes. Nesse sentido, o Núcleo Ampliado de Saúde da Família e Atenção Básica (NASF-AB) faz parte do primeiro contato, onde o Fisioterapeuta atua com estratégias de prevenção e reabilitação para os usuários, o que evidencia sua importância na reabilitação após um AVE. Sua conduta do atendimento domiciliar junto com a equipe da UBS traz inúmeros benefícios para esses usuários (PAUFERRO., et al 2018).

Além disso, outras enfermidades fazem parte da população acima dos 60 anos, entre elas destaca-se a incontinência urinaria, que dificulta as Atividades de Vida Diária (AVD) dosidosos. A incontinência urinária pode ser de urgência, esforço ou mista e afeta diretamente o meio social do indivíduo pelo desconforto de não controlar o ato miccional (DOS SANTOS; VAZ, 2017).

Diante disso, o profissional responsável por reabilitar a incontinência urinaria é o fisioterapeuta com estratégias de fortalecimento dos músculos do assoalho pélvico e reeducação dos músculos abdominais, além de exercícios ativos que ajudam diretamente nas AVD deste paciente que na sua grande maioria são os idosos que frequentam a AP (FREITAS., et al 2020).

Ademais, enfermidades como essas estão sempre presentes na UBS, e a equipe multiprofissional busca intervir de forma satisfatória para que seja uma resolutividade na condição de saúde trazida pelo usuário, sendo evidente que o fisioterapeuta exerce um papel fundamental para promover estratégia preventivas como as de orientação reabilitação para a população idosa (BERNADES; NUNES, 2021).

Outrossim, é a dor lombar crônica que afeta o adulto jovem e mais ainda o idoso, seja por posturas inadequadas adotadas ao longo de sua vida ou pelo ambiente de trabalho ergonomicamente incorreto, desfavorecendo o cuidado com as estruturas da coluna vertebral. As táticas de prevenção são feitas na maioria das 
vezes de orientações por meio de salas de esperas, escuta inicial, acolhimento que são realizadas pela equipe multiprofissional na AP (MENDONÇA., et al 2017).

Os distúrbios musculoesqueléticos na idade avançada estão sempre presentes e precisam de um profissional capacitado para tratar destes pacientes, como por exemplo, o fisioterapeuta se adequa perfeitamente para trabalhar na AP este profissional pode desenvolver atividades emgrupos para tratar distúrbios em comum como também atua individualmente de acordo com cada doença (DANTAS., et al 2020).

Outro acometimento de grande importância que merece uma atenção especial entre as doenças crônicas que afetam diretamente a vida dos idosos são as artroses, osteoporose e a artrite reumatoide que apresentando grau avançado podem dificultar a locomoção e as AVD deste idosos. Contudo, estudos apontam que por meio da prática de atividades físicas, o corpo é capaz de diminuir a degeneração destas ocasionada por essas doenças. Além disso, se essas atividades forem por meio de grupos podem ajudar a interação e diminuição da depressão nestaidade (SEUS., et al 2019).

As abordagens em grupos têm sido benéficas para os usuários da $A P$, pois abrangem um público com doenças crônicas em comum que juntos recebem uma assistência qualificada e acolhedora para todos os pacientes, estando em ênfase os idosos, que são em grande parte hipertensos e/ou diabéticos (POBLETE., et al 2018).

Portanto, como proposta de melhorias, a AP pode adotar abordagens voltadas para o bem-estar físico e social dos usuários que frequentam as UBS, que podem ser realizadas por meio de orientações, escuta inicial, grupos da melhor idade, acolhimento entre outras estratégias com desfechos para a resolução dos problemas destes pacientes, pois a AP pode contar com a equipe multiprofissional para melhorias de ações e prevenções da melhor idade (DA FONSECA., et al 2016). 


\section{CONCLUSÃO}

Diante deste contexto, o fisioterapeuta é capacitado para realizar uma reabilitação resolutiva de acordo com cada individualidade do paciente idoso na AP, este faz a diferença, pois conta com a equipe multiprofissional para dar um proposito frutífero para as questões de saúde trazidas pelos usuários, sendo evidente destacar que o fisioterapeuta executa suasestratégias correspondente para a maioria dos seus usuários frequentes da $\mathrm{AP}$, que são os idosos, e traz consigo condutas em grupos e individuais resolutivas dentro de uma UBS fazendo a diferença na vida dos idosos.

\section{REFERÊNCIAS BIBLIOGRÁFICAS}

ANDERSSON, Siv Folkhammar et al. Arthritis management in primary care-A study of physiotherapists' current practice, educational needs and adherence to national guidelines. Musculoskeletal care, v. 15, n. 4, p. 333-340, 2017.

BERNARDES, Thábita Vilarinho; NUNES, Marilene Rivany. Prevalência do idoso em situação de fragilidade na atenção primária a saúde. Brazilian Journal of Development, v. 7, n. 1, p. 9031-9040, 2021.

BRASIL. Serviço de Atenção Domiciliar Bahia (SAD-BA). Serviços de atenção domiciliar: padronização, fluxos e rotinas técnicas. Disponível em: http://www.pbh.gov.br/smsa/biblioteca/geas/assistenciadomiciliar.pdf. Acesso em: 08/01/2021.

CARDOSO, Alex Dos Santos; DE MELLO, Alessandro Pedrosa Vieira. INTERVENÇÃO DA FISIOTERAPIA NA ESTRATÉGIA DA SAÚDE DA FAMÍLIA (ESF) NA SAÚDE DO IDOSO. HÍGIA-REVISTA DE CIÊNCIAS DA SAÚDE E SOCIAIS APLICADAS DO OESTE BAIANO, v. 5, n. 1, 2020.

DA FONSECA, Juliany Marques Abreu et al. A Fisioterapia na Atenção Primária à Saúde: uma revisão integrativa. Revista Brasileira em Promoção da Saúde, v. 29, n. 2, p. 288-294,2016.

DANTAS, Bruno A. da S. et al. Impact of multidimensional interventions on quality of life and depression among older adults in a primary care setting in Brazil: a quasi-experimental study. Brazilian Journal of Psychiatry, v. 42, n. 2, p. 201-208, 2020.

DE LIMA, Ana Jéssica et al. RESOLUTIVIDADE DA FISIOTERAPIA NA ATENÇÃO BÁSICA À SAÚDE (AB): A PERCEPÇÃO DE FISIOTERAPEUTAS. CADERNOS DEEDUCAÇÃO, SAÚDE E FISIOTERAPIA, v. 4, n. 8, 2017.

DE OLIVEIRA, Beatriz Campos et al. Avaliação da qualidade de vida em idosos dacomunidade. Revista Brasileira em Promoção da Saúde, v. 30, n. 3, 2017. 
DÍAZ-CERRILLO, Juan Luis et al. Ensayo no aleatorizado de una intervención educativa basada en principios cognitivo-conductuales para pacientes con lumbalgia crónica inespecífica atendidos en fisioterapia de atención primaria. Atención Primaria, v. 48, n. 7, p.440-448, 2016.

DOS SANTOS, Carine Sousa et al. Capacidade funcional de idosos acompanhados pela Fisioterapia de uma Unidade de Atenção Primária à Saúde. Revista FisiSenectus, v. 7, n. 2,p. 23-38, 2019.

DOS SANTOS, Ruth Ellen Ribeiro; VAZ, Camila Teixeira. Conhecimento de profissionais da atenção primária à saúde sobre a abordagem terapêutica na incontinência urinária feminina. HU Revista, v. 43, n. 3, p. 239-245, 2017.

FREITAS, Crislainy Vieira et al. Abordagem fisioterapêutica da incontinência urinária em idosos na atenção primária em saúde. Fisioterapia e Pesquisa, v. 27, n. 3, p. 264-270, 2020.

KINGE, Jonas Minet et al. Musculoskeletal disorders in Norway: prevalence of chronicity and use of primary and specialist health care services. BMC musculoskeletal disorders, v.16, n. 1, p. 1-9, 2015.

MEDEIROS, M. A. D.; PAIXÃO, M.C.; MIRANDA, L.N. Atenção multidisciplinar através do Consultório na Rua. Ciências Biológicas e de Saúde Unit, Alagoas, 2017; v. 4, n. 2, p.283296.

MENDONÇA, Francielle Toniolo Nicodemos Furtado de et al. Educação em saúde comidosos: pesquisa-ação com profissionais da atenção primária. Revista Brasileira de Enfermagem, v. 70, n. 4, p. 792-799, 2017.

MONTENEGRO, Pedrita da Cunha Sales Pereira; BARBOSA, Inara Sol; NOVAES, Maria Rita Carvalho Garbi. Avaliação geriátrica em um serviço da Atenção Primária à Saúde: riscos e propostas de intervenção. Comunicação em Ciências da Saúde, 2020.

PAUFERRO, Ana Luíza Moreira et al. O trabalho de fisioterapeutas de Núcleos de Apoio à Saúde da Família na assistência ao idoso. Revista Família, Ciclos de Vida e Saúde no Contexto Social, v. 6, p. 571-580, 2018.

PIUVEZAM, Grasiela et al. Atenção primária à saúde e os idosos institucionalizados: a perspectiva da gestão municipal no Brasil. Revista Portuguesa de Saúde Pública, v. 34, n. 1, p. 92-100, 2016.

PLACIDELI, Nádia et al. Avaliação da atenção integral ao idoso em serviços de atenção primária. Revista de Saúde Pública, v. 54, p. 06, 2020.

POBLETE, Fernando et al. Efectividad de un modelo de manejo de pacientes con hipertensión y diabetes tipo II en atención primaria. Revista médica de Chile, v. 146, n. 11, p. 1269-1277, 2018.

SEUS, Thamires Lorenzet Cunha et al. Núcleo de Apoio à Saúde da Família: promoção da saúde, atividade física e doenças crônicas no Brasil-inquérito nacional PMAQ 2013. Epidemiologia e Serviços de Saúde, v. 28, p. e2018308, 2019.

SILVA, Laize Gabriele de Castro et al. Avaliação da funcionalidade e mobilidade de idosos comunitários na atenção primária à saúde. Revista Brasileira de Geriatria e Gerontologia,v. 22, n. 5, 2019.

TOMASI, Andrelise Viana Rosa et al. Incontinência urinária em idosas: práticas assistenciaise proposta de cuidado âmbito da atenção primária de saúde. Texto \& contexto enferm [Internet], v. 26, n. 2, p. 1-10, 2017. 
TORRES, Ruth Cristini et al. Perfil de idosos hipertensos e diabéticos de um município de Sergipe. Saúde Coletiva (Barueri), v. 10, n. 59, p. 4376-4387, 2020.

ØSTERÅS, Nina et al. Implementing a structured model for osteoarthritis care in primary healthcare: A stepped-wedge cluster-randomised trial. PLoS medicine, v. 16, n. 10, p. e1002949, 2. 